\title{
TOPOLOGY OF INTERCONNECTION NETWORKS WITH GIVEN DEGREE AND DIAMETER
}

\author{
GUILLERMO PINEDA-VILLAVICENCIO
}

(Received 10 September 2009)

2000 Mathematics subject classification: primary 05C50, 05C75, 05C76, 90C27; secondary 68M10, $90 \mathrm{~B} 18$.

Keywords and phrases: graph, interconnection network.

This thesis deals with the design of optimal interconnection networks. Optimality is interpreted as the largest possible number of nodes in the network, under given constraints on the number of connections attached to a node (degree of the node), and on the length of shortest paths between any two nodes (diameter of the network). Any interconnection network with bidirectional channels can be modelled by an undirected graph referred to as the 'topology' of the network. In graph theory this interpretation of optimality is known as the degree/diameter problem [8]. Under these constraints, there is an upper bound $M_{\Delta, D}$ on the maximum number of nodes that a network of maximum degree $\Delta$ and diameter $D$ can have, which is known as the Moore bound [2,8]. For bipartite network topology, the bipartite Moore bound $M_{\Delta, D}^{b}$ is defined in a similar way to the general Moore bound $[2,8]$.

I address the degree/diameter problem for both general graphs and bipartite graphs. Interconnection networks modeled by (bipartite) Moore graphs-namely, (bipartite) graphs attaining the (bipartite) Moore bound-are optimal.

Studies on Moore graphs and bipartite Moore graphs proved their rarity [1, 6, 12]. Consequently, research efforts to search for such optimal graphs fall into two main streams.

(i) Lowering the upper bound on the number of vertices of a graph of maximum degree $\Delta$ and diameter $D$ by proving the nonexistence or otherwise of graphs whose number of vertices is close to the corresponding Moore bounds.

(ii) Increasing the lower bound on the number of vertices of a graph of maximum degree $\Delta$ and diameter $D$ by constructing largest known graphs of maximum degree $\Delta$ and diameter $D$.

Thesis submitted to the University of Ballarat, January 2009. Degree approved, May 2009. Supervisors: Professor Mirka Miller, University of Newcastle and Professor John Yearwood, University of Ballarat.

(C) 2010 Australian Mathematical Publishing Association Inc. 0004-9727/2010 \$16.00 
(Bipartite) graphs of given maximum degree $\Delta$, diameter $D$ and order $\left(M_{\Delta, D}^{b}-\epsilon\right)$ $M_{\Delta, D}-\epsilon$ are called (bipartite) $(\Delta, D,-\epsilon)$-graphs.

I also investigate regular topologies with the smallest possible number of vertices given degree and girth (the length of a shortest cycle in the graph): the degree/girth problem [5]. I consider only the case of even girth, in which case the bipartite Moore bound is the best known lower bound on the minimum number of vertices of such topologies. Regular graphs of degree $d$, girth $g$ and order $M_{d, g / 2}^{b}+\epsilon$ are called $(d, g,+\epsilon)$-graphs.

The following results are the cornerstones of this thesis.

Large graphs of diameter 6. I produce a family of large compound graphs of diameter 6 (see [10]). Several members of this family are currently the largest known graphs for their respective maximum degree.

Complete catalogue of $(3, D,-4)$-graphs. In the context of the degree/diameter problem for general graphs, I complete the characterization of all $(3, D,-\epsilon)$ graphs with $D \geq 2$ and $0 \leq \epsilon \leq 4$, the main result being a nonexistence proof of (3, $D,-4)$-graphs for $D \geq 5$ (see $[7,11])$.

Bipartiteness of $(3, g,+4)$-graphs for even $\boldsymbol{g}$. I study the degree/girth problem for even girth, obtaining as the main result that if $(3, g,+4)$-graphs with $g \geq 12$ exist then they must be bipartite. Furthermore, I conjecture that such graphs do not exist.

Bipartite $(\Delta, 3,-2)$-graphs. I study the degree/diameter problem for bipartite graphs. More precisely, I derive several necessary conditions for the existence of bipartite $(\Delta, 3,-2)$-graphs, and prove the uniqueness of the two known such graphs (see [4]).

Bipartite $(\boldsymbol{\Delta}, \boldsymbol{D},-\mathbf{- 2})$-graphs. This research follows up previous studies concerning the bipartite version of the degree/diameter problem. Here I prove that bipartite ( $\Delta, D,-2)$-graphs with $\Delta \geq 3$ and $D \geq 4$ do not exist (see [3, 9]).

Finally, I present a number of open problems and conjectures, providing scope for future research in the area of network design.

\section{References}

[1] E. Bannai and T. Ito, 'On finite Moore graphs', J. Math. Sci. Univ. Tokyo 20 (1973), 191-208.

[2] N. L. Biggs, Algebraic Graph Theory, 2nd edn (Cambridge University Press, Cambridge, 1993).

[3] C. Delorme, L. K. Jørgensen, M. Miller and G. Pineda-Villavicencio, 'On bipartite graphs of defect 2', European J. Combin. 30(4) (2009), 798-808.

[4] C. Delorme, L. K. Jørgensen, M. Miller and G. Pineda-Villavicencio, 'On bipartite graphs of diameter 3 and defect 2', J. Graph Theory 61(4) (2009), 271-288.

[5] G. Exoo and R. Jajcay, 'Dynamic cage survey', Electron. J. Combin. (2008), 1-48. Dynamic survey DS16.

[6] W. Feit and G. Higman, 'The nonexistence of certain generalized polygons', J. Algebra 1 (1964), $114-131$. 
[7] M. Miller and G. Pineda-Villavicencio, 'Complete catalogue of graphs of maximum degree 3 and defect at most 4', Discrete Appl. Math. 157(13) (2009), 2983-2996.

[8] M. Miller and J. Širáň, 'Moore graphs and beyond: a survey of the degree/diameter problem', Electron. J. Combin. (2005), 1-61. Dynamic survey DS14.

[9] G. Pineda-Villavicencio, 'Non-existence of bipartite graphs of diameter at least 4 and defect 2', submitted.

[10] G. Pineda-Villavicencio, J. Gómez, M. Miller and H. Pérez-Rosés, 'New largest known graphs of diameter 6', Networks 53(4) (2009), 315-328.

[11] G. Pineda-Villavicencio and M. Miller, 'On graphs of maximum degree 3 and defect 4', J. Combin. Math. Combin. Comput. 65 (2008), 25-31.

[12] R. C. Singleton, 'On minimal graphs of maximum even girth', J. Combin. Theory 1(3) (1966), 306-332.

GUILLERMO PINEDA-VILLAVICENCIO, Centre for Informatics and Applied

Optimization, University of Ballarat, University Drive, Mount Helen, Victoria 3353, Australia

e-mail: work@guillermo.com.au 